\title{
Evaluation of effectiveness and efficiency of wild bird surveillance for avian influenza
}

\author{
Theodore J.D. Knight-Jones ${ }^{1 *}$, Ruth Hauser ${ }^{2}$, Doris Matthes ${ }^{3}$, \\ Katharina D.C. STÄRK ${ }^{1}$ \\ ${ }^{1}$ The Royal Veterinary College, University of London, Wardens house, Hawkshead lane, North Mymms, Herts., \\ AL9 7TA, United Kingdom \\ ${ }^{2}$ Swiss Federal Veterinary Office, Bern, Switzerland \\ ${ }^{3}$ Max Planck Institute for Ornithology, Radolfzell, Germany
}

(Received 9 December 2009; accepted 19 April 2010)

\begin{abstract}
This study aimed to assess which method of wild waterbird surveillance had the greatest probability of detecting highly pathogenic avian influenza (HPAI) H5N1 during a period of surveillance activity, the cost of each method was also considered. Lake Constance is a major wintering centre for migratory waterbirds and in 2006 it was the site of an HPAI H5N1 epidemic in wild birds. Avian influenza surveillance was conducted using harmonised approaches in the three countries around the lake, Austria, Germany and Switzerland, from 2006-2009. The surveillance consisted of testing birds sampled by the following methods: live birds caught in traps, birds killed by hunters, birds caught in fishing nets, dead birds found by the public and catching live Mute Swans (Cygnus olor); sentinel flocks of Mallards (Anas platyrhynchos) were also used. Scenario tree analysis was performed including sensitivity analysis, followed by assessment of cost-effectiveness. Results indicated that if HPAI H5N1 was present at $1 \%$ prevalence and assuming HPAI resulted in bird mortality, sampling dead birds found by the public and sentinel surveillance were the most sensitive approaches despite residual uncertainty over some parameters. The uncertainty over the mortality of infected birds was an influential factor. Sampling birds found dead was most cost-effective, but strongly dependent on mortality and awareness of the public. Trapping live birds was least cost-effective. Based on our results, we recommend that future HPAI H5N1 surveillance around Lake Constance should prioritise sentinel surveillance and, if high mortality is expected, the testing of birds found dead.
\end{abstract}

\section{scenario tree analysis / surveillance / avian influenza / wild bird / cost-effectiveness}

\section{INTRODUCTION}

The threat posed to human health by zoonotic influenza viruses has been a cause for great concern [6]. In addition, outbreaks of highly pathogenic avian influenza (HPAI) have a big impact on the health of domestic poultry worldwide and have required costly control measures [2].

\footnotetext{
* Corresponding author: tkjones@rvc.ac.uk
}

Compared to other birds, waterbirds are commonly infected with avian influenza (AI) viruses [2, 22, 23]. HPAI subtype H5N1, unlike other subtypes, causes mortality in wild birds and there is a concern that it may be sufficiently maintained within wild bird populations to allow long distance transmission from wild birds to poultry, although the evidence is unclear on this latter point $[2,19]$. Migratory waterbirds in particular are thought to play a key but poorly understood role in the transmission of HPAI H5N1. Transmission within the

This is an Open Access article distributed under the terms of the Creative Commons Attribution-Noncommercial License (http://creativecommons.org/licenses/by-nc/3.0/), which permits unrestricted use, distribution, and reproduction in any noncommercial medium, provided the original work is properly cited. 
poultry sector (without the involvement of wild birds) is also important $[1,16]$.

In Europe, wild birds were heavily involved in HPAI H5N1 outbreaks in 2006 with 861 wild bird cases and suspected epidemiological links of wild birds to many of the 205 outbreaks in domestic birds [7]. Austria, Germany and Switzerland were among the countries affected, with 86 infected wild birds found near Lake Constance [9], which is surrounded by these three countries.

Lake Constance is an important migratory waterbird site, with over 200000 waterbirds present during winter. To aid wild bird AI surveillance in the area, a tri-national cooperative programme was initiated (the Constanze Project $\left.{ }^{1}\right)$. Within this programme, six different Surveillance System Components (SSC) were used, each using a different method to sample waterbirds.

Due to competing demands for limited resources, there is a need to identify optimal approaches to surveillance. Evaluation of a SSC cannot be based on its ability to detect outbreaks alone, the cost must also be considered.

The sensitivity of a SSC is the probability that it detects the disease or infection of interest when it is present in the population under surveillance. The aims of this study were to apply scenario tree analysis to estimate and compare the sensitivity of the different SSC under particular conditions, to identify important parameters that influence sensitivity and to assess the cost-effectiveness of the SSC. Only HPAI $\mathrm{H} 5 \mathrm{~N} 1$ surveillance of wild waterbirds was considered.

\section{MATERIALS AND METHODS}

Scenario tree analysis is a quantitative methodology that has been used for analysing complex veterinary surveillance systems since it was developed in 2007 [17, 18]. In this method, trees are developed to describe the chain of events that must occur for a bird to become infected and detected by a SSC.

\footnotetext{
${ }^{1}$ Swiss Federal Veterinary Office, Constanze Project, [on line] www.bvet.admin.ch/gesundheit_tiere/ 00276/00280/index.html [consulted 6 December 2009].
}

Each event is represented by a node in the tree with more than one possible outcome. For each node a probability is given for each possible outcome. These probabilities are obtained from data, literature or expert opinion. The probabilities are then combined to estimate the sensitivity of the SSC. This was done for each of the six methods of sampling wild birds considered in this study (see below).

Cost-effectiveness relates the performance of public health interventions to their cost [12]. Providing the ratio of the direct cost of a SSC to the sensitivity (i.e. cost per $1 \%$ sensitivity) provides a valuable measure of efficiency by which to compare SSC.

Abbreviations used are shown in Table I.

\subsection{Surveillance system components}

Within the Constanze Project, six different methods of sampling wild birds to then test for avian influenza were implemented at Lake Constance.

(a) Bycatch: Waterbirds accidentally caught in commercial fishing nets were submitted to the veterinary authorities.

(b) Live trapping (Trap): Two traps positioned at strategic locations around the lake were used to catch live waterbirds, one in Switzerland and one in Germany.

(c) Catching of live swans (Swan Catch): During the moulting period, live Mute Swans (Cygnus olor) were caught (August-September). During the moult, swans lose their flight feathers and are unable to fly making them easier to catch.

(d) Found dead: Birds found dead and reported by members of the public were collected. No active patrolling for dead birds was performed during the period of study.

(e) Hunted (Hunt): Some birds killed by hunters were sampled.

(f) Sentinel surveillance (Sentinel): Three separate flocks of approximately 12 sentinel Mallards (Anas platyrhyncos) were kept, close contact between sentinel and wild birds was possible. Sentinel birds were sampled fortnightly.

\subsection{Sampling and laboratory tests}

Tracheal and cloacal swabs were taken from all birds and analysed as described by Globig et al. [8]. Briefly, RT-PCR tests were performed in series to confirm if a sample was HPAI H5N1 virus positive. Sequencing was attempted on H5 positive 
Table I. Glossary of abbreviations.

\begin{tabular}{|c|c|}
\hline Abbreviation & Explanation \\
\hline $\mathrm{SSC}$ & Surveillance System Component \\
\hline$D / G / M$ & Bird group "Diving ducks, Grebes and Mergansers" \\
\hline $\operatorname{Pr}_{-} i$ & $\begin{array}{l}\text { Proportion of birds within bird group } i \text {, where } i \text { represents Swan, } \\
D / G / M \text { or Other }\end{array}$ \\
\hline$P^{*} u$ & Probability that a bird was infected (Design Prevalence) \\
\hline P_Dies_i & $\begin{array}{l}\text { Probability that a bird died given it was infected, } \\
\text { where } i \text { represents the Bird group }\end{array}$ \\
\hline TestSe_Dead or TestSe_Live & Sensitivity of diagnostic tests for dead and live birds respectively \\
\hline PSamp_ $i$ & $\begin{array}{l}\text { Proportion of birds sampled for each bird group, } \\
\text { where } i \text { represents the Bird group }\end{array}$ \\
\hline$N \_S a m p \_i$ & Number of birds sampled (Hunt and Found dead) \\
\hline Surv_i & Normal yearly survival for each bird group \\
\hline SeSent_i & $\begin{array}{l}\text { Probability of detecting an infected bird by sentinel } \\
\text { surveillance given it had not died, } \\
\text { where } i \text { represents the Bird group }\end{array}$ \\
\hline PrInfect & $\begin{array}{l}\text { Proportion of sentinel birds infected at the time of } \\
\text { testing given that the flock was infected }\end{array}$ \\
\hline PInf_ij & $\begin{array}{l}\text { Probability that an infected wild bird visitor infected } \\
\text { the sentinel flock, where } i \text { represents Bird group } \\
\text { and } j \text { represents Expert } 1 \text { or Expert } 2\end{array}$ \\
\hline CSe_i & Component sensitivity, where $i$ represents the different SSC \\
\hline $\operatorname{Pr} \bar{j}$ & Proportion of "Other" birds that are "Mallard" or "Non_Mallard" \\
\hline PVisit_i & $\begin{array}{l}\text { Proportion of birds that visited the sentinel flocks, } \\
\text { where } i \text { represents the Bird group }\end{array}$ \\
\hline FlockTestse & Diagnostic test flock level sensitivity for a sentinel flock \\
\hline Cost_i & Cost of surveillance, where $i$ represents the different SSC \\
\hline$S R$ & Sensitivity ratio \\
\hline
\end{tabular}

samples. The suitability of serological tests of sentinel birds for HPAI was unclear, they were therefore not considered. Tests were conducted by laboratories routinely involved in AI surveillance in the regions around the lake in the three countries. Test results were obtained from the Constanze Project database, maintained by the Friedrich-Loeffler-Institute, Insel Riems, Germany.

\subsection{Scenario tree models}

In order to estimate the sensitivity of the different SSC, scenario trees were created [18]. In all trees, the first node (Bird group) describes how the wild bird population was split into different groups; Swans (Cygnus sp.) in one group, diving ducks (Netta sp. and Aythya sp.), grebes (Tachybaptus sp. and Podiceps sp.) and mergansers (Mergus sp.) as another group (referred to as $D / G / M$ ) and all remaining waterbirds were grouped as Other (see Tab. II). These groupings were based on differences in factors that affect detection between the bird groups. The next two nodes for all trees represent the design prevalence (Unit infection status), followed by whether or not the infected bird died (Bird dies). Except for sentinel, the subsequent nodes represent the bird being sampled and correctly testing positive, see Figures 1 and 2.

For sentinel surveillance, after the node Bird dies, the bird group "Other" was further categorised as Mallard (Anas platyrhyncos) or NonMallard. Subsequent nodes represent the bird visiting the sentinel, infecting the sentinel flock and this infection being detected, see Figure 3.

Because wild bird populations vary over the year, particularly for migratory birds, trees were parameterised to represent an average month from MayAugust 2007 as well as for an average month from September 2006-April 2007; months were grouped to represent periods when these differences were 
Table II. Scenario tree node values for the different Surveillance System Components (SCC) used for HPAI H5N1 surveillance in wild waterbirds on Lake Constance. Branch probabilities are either a single point value or a distribution; @RISK distributions are shown when appropriate, otherwise the expected value is shown. Where applicable, branch probabilities are shown for the different wild waterbird groups: diving ducks, grebes and mergansers as one group $(D / G / M)$, Swans and Other. The relevant scenario trees are shown in Figures 1-3.

\begin{tabular}{|c|c|c|c|}
\hline Tree & Node name & Branch name & Branch probability \\
\hline All SSC: May-August & Bird group & $\begin{array}{l}\text { Swans } \\
D / G / M \\
\text { Other }\end{array}$ & $\begin{array}{c}0.0177 \text { (number of birds 208) } \\
0.189 \text { (number of birds } 2943 \text { ) } \\
0.797 \text { (number of birds } 12633,16 \% \text { dabbling } \\
\text { ducks and } 58 \% \text { Gulls (Laridae)) }\end{array}$ \\
\hline All SSC: September-April & Bird group & $\begin{array}{l}\text { Swans } \\
D / G / M \\
\text { Other }\end{array}$ & $\begin{array}{c}0.0143 \text { (number of birds } 1912 \text { ) } \\
0.541 \text { (number of birds } 72620 \text { ) } \\
0.444 \text { (number of birds } 59525,30 \% \\
\text { dabbling ducks, } 56 \% \text { Eurasian Coots (Fulica atra)) }\end{array}$ \\
\hline All trees & Unit infection status & Infected & 0.01 \\
\hline All trees & Bird dies & Dies & $\begin{array}{c}\text { Swan } \sim \text { Uniform }(0.8,0.99) ; D / G / M \sim \text { Uniform }(0.2,0.8 \\
\text { Other } \sim \text { Uniform }(0.2,0.6)\end{array}$ \\
\hline $\begin{array}{l}\text { Bycatch, Swan Catch, } \\
\text { Hunt \& Trap }\end{array}$ & Test positive & Yes & BetaPert $(0.7,0.8,0.9)-$ for live birds \\
\hline Found dead & Test positive & Yes & Uniform $(0.9,0.99)$ - for dead birds \\
\hline September-April trees & & & \\
\hline $\begin{array}{l}\text { Bycatch } \\
\text { Trap }\end{array}$ & $\begin{array}{l}\text { Bird sampled } \\
\text { Bird sampled }\end{array}$ & $\begin{array}{l}\text { Sampled } \\
\text { Sampled }\end{array}$ & $\begin{array}{c}\text { Swan }=0 ; D / G / M=1.89 \mathrm{E}^{-5} ; \text { Other }=2.31 \mathrm{E}^{-5} \\
\text { Swan }=3.27 \mathrm{E}^{-4} ; D / G / M=6.20 \mathrm{E}^{-5} \\
\text { Other }=4.82 \mathrm{E}^{-5}\end{array}$ \\
\hline Swan Catch & Bird sampled & Sampled & Swan $=0.197 ; D / G / M=0 ;$ Other $=0$ \\
\hline Found dead & Bird sampled & Sampled & $\begin{array}{c}\text { Expected values: Swan }=0.0123 \text {; } \\
D / G / M=1.46 \mathrm{E}^{-4} ; \text { Other }=5.23 \mathrm{E}^{-4}\end{array}$ \\
\hline Hunt & Bird sampled & Sampled & $\begin{array}{l}\text { Expected values: Swan }=9.81 \mathrm{E}^{-4} ; \\
D / G / M=5.16 \mathrm{E}^{-5} ; \text { Other }=5.24 \mathrm{E}^{-5}\end{array}$ \\
\hline
\end{tabular}


Table II. Continued.

\begin{tabular}{|c|c|c|c|}
\hline Tree & Node name & Branch name & Branch probability \\
\hline \multirow{4}{*}{ Sentinel } & Mallards & Yes & 0.154 (number of Mallard ducks 9 206) \\
\hline & Bird visits sentinel & Visits & $\begin{array}{c}\text { Expected values: Swan }=0.003 ; D / G / M=0 \text { : } \\
\text { Non_Mallard }=5.0 \mathrm{E}^{-4} ; \text { Mallard }=0.0348\end{array}$ \\
\hline & Infects sentinel & Yes & $\bar{S}$ wan $\sim$ Betapert $(0.2,0.5,0.8)$ or \\
\hline & $\begin{array}{l}\text { Expert } 1 \text { or } \\
\text { Expert } 2\end{array}$ & & $\begin{array}{c}\text { Betapert }(0.2,0.7,1) ; D / G / M \sim \text { Betapert }(0.1,0.2,0.5) \text { or } \\
\text { BetaPert }(0.2,0.4,1) ; \text { Other_Non_Mallard } \sim \text { Betapert } \\
(0.1,0.2,0.5) \text { or Betapert }(0.2,0.6,1) ; \\
\text { Other_Mallard } \sim \text { Betapert }(0.2,0.5,1) \text { or Betapert } \\
(0.2,0.9,1)\end{array}$ \\
\hline
\end{tabular}

\section{Flock test positive}

May-August trees

Bycatch

Trap

Swan Catch

Found dead

\section{Hunt}

Sentinel

Bird sampled
Bird sampled
Bird sampled

Bird sampled

Bird sampled

Mallard

Bird visits sentinel

Infects sentinel

Flock test positive
Yes

Sampled

Sampled

Sampled

Sampled

Sampled

Yes

Visits

Yes
Expected value: 0.999

$$
\begin{gathered}
\text { Swan }=0 ; D / G / M=0 ; \text { Other }=1.98 \mathrm{E}^{-5} \\
\text { Swan }=2.68 \mathrm{E}^{-3} ; D / G / M=8.49 \mathrm{E}^{-4} ; \\
\text { Other }=1.35 \mathrm{E}^{-3}
\end{gathered}
$$

Swan $=6.08 \mathrm{E}^{-3} ; D / G / M=0 ;$ Other $=6.29 \mathrm{E}^{-6}$

Expected value: Swan $=0.0714 ; D / G / M=3.80 \mathrm{E}^{-3}$; Other $=5.45 \mathrm{E}^{-3}$

Swan $=0 ; D / G / M=0 ;$ Other $=0$

0.150 (number of Mallard ducks 1898 )

Expected value: Swan $=0 ; D / G / M=0$; NonMallard $=1.4 \mathrm{E}^{-3} ;$ Mallard $=0.12$

As for sentinel September-April

As for sentinel September-April 


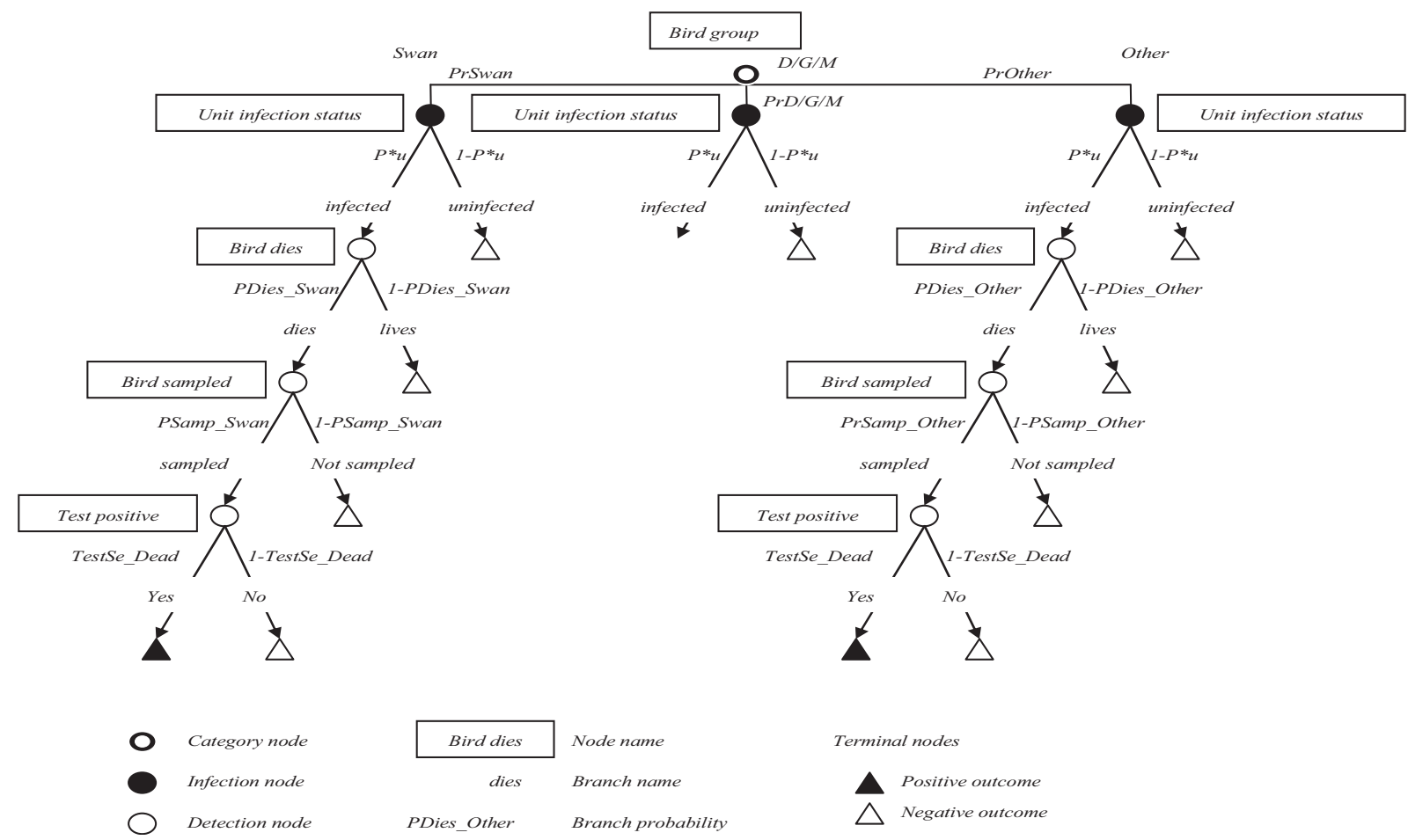

Figure 1. Scenario tree for the surveillance of HPAI H5N1 in wild waterbirds on Lake Constance by sampling birds Found dead. Branch for $D / G / M$ is not completed but would have the same structure as the branch for Swan. 


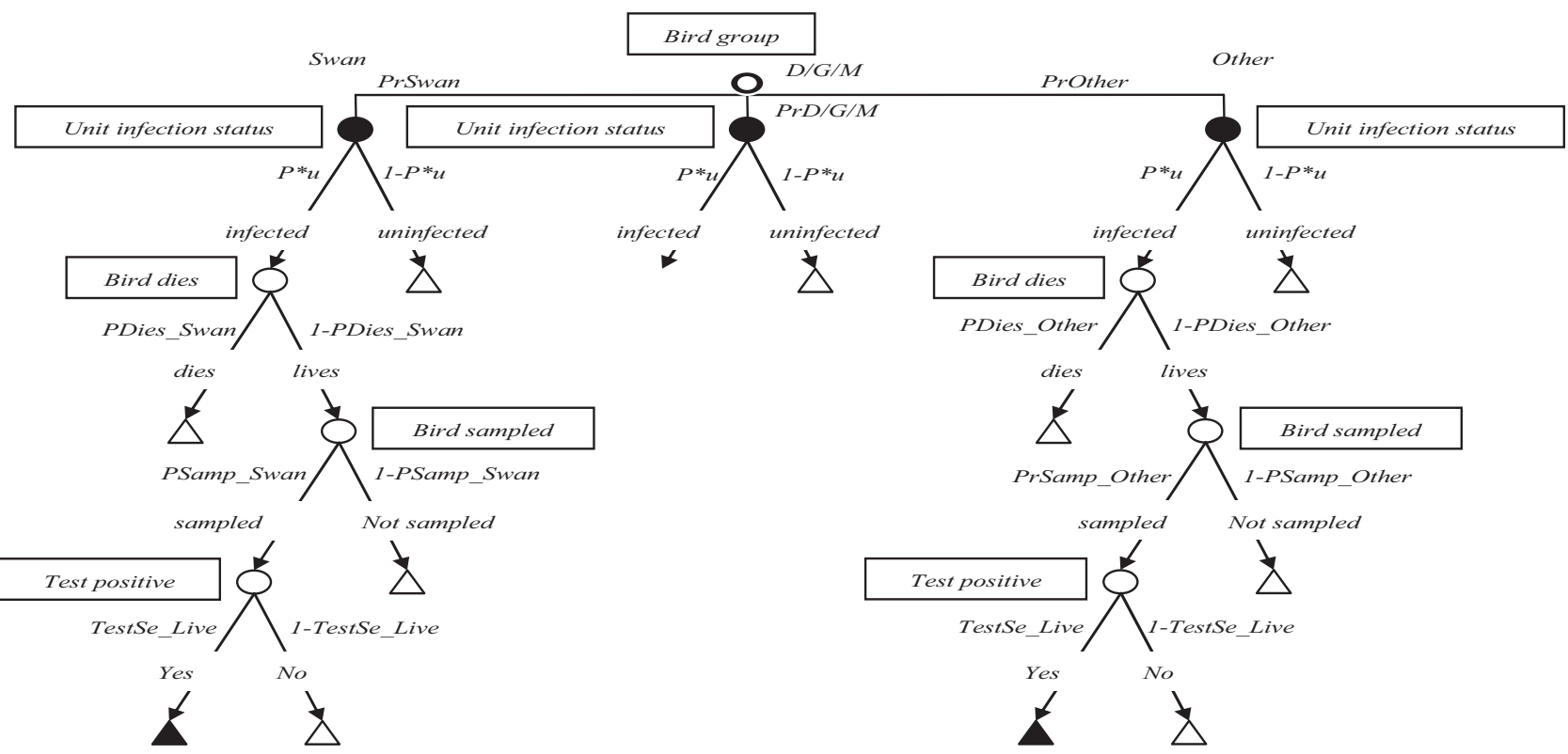

Figure 2. Scenario tree for the surveillance of HPAI H5N1 in wild waterbirds on Lake Constance by sampling live birds i.e. sampling birds by Bycatch, Trap, Swan Catch or Hunt. Branch for $D / G / M$ is not completed but would have the same structure as the branch for Swan. 


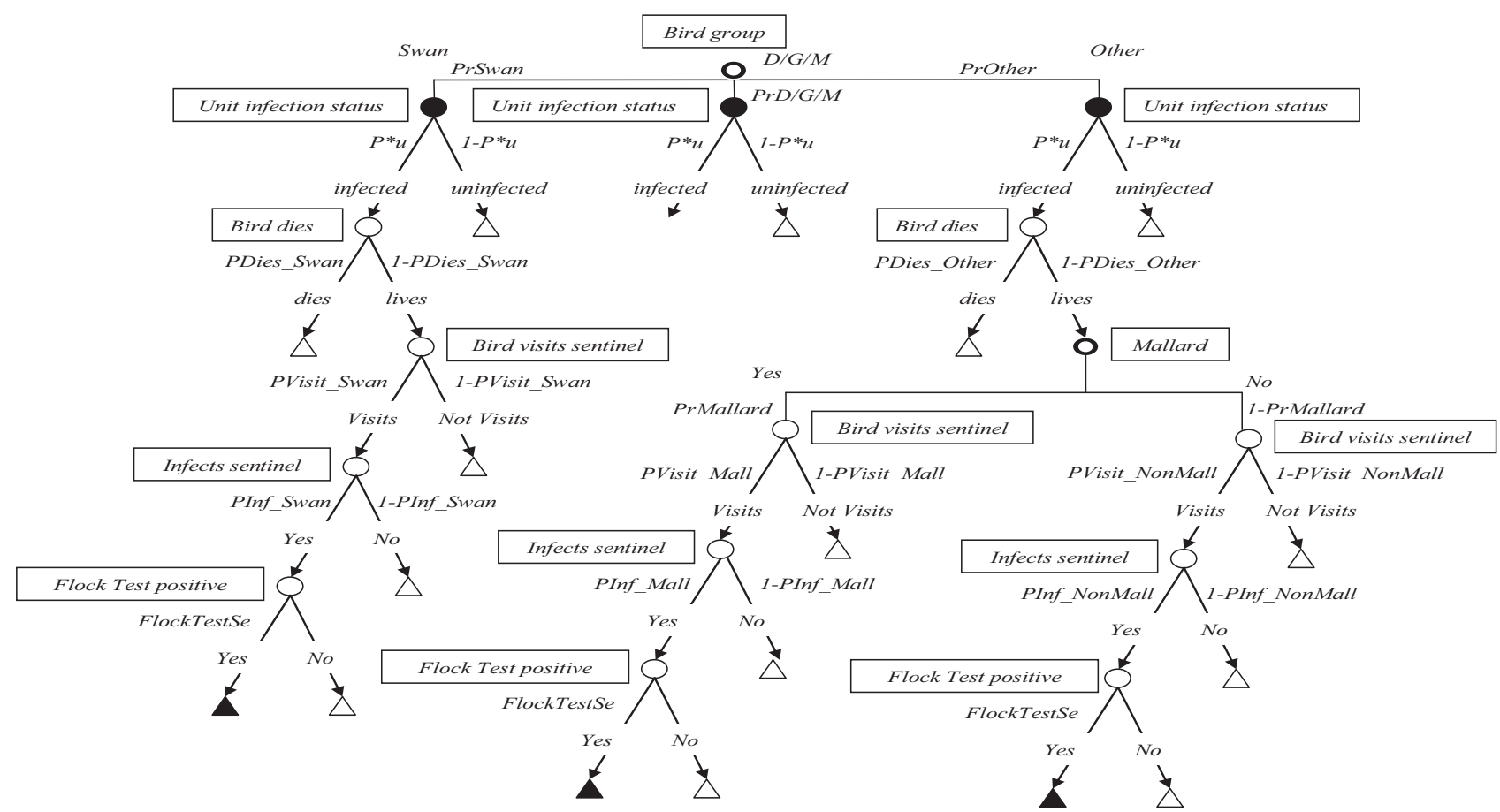

Figure 3. Scenario tree for the surveillance of HPAI H5N1 in wild waterbirds on Lake Constance using sentinel flocks of Mallard ducks (Anas platyrhynchos). Branch for $D / G / M$ is not completed but would have the same structure as the branch for Swan. 
most pronounced. Values that showed seasonal variation included numbers and proportions present for each bird group and the proportion of birds that were sampled or that visited sentinel flocks.

\subsection{Node values}

Nodes in a scenario tree represent events that must occur for infection and subsequent detection of disease, or they describe how the population is split into different groups with differing probabilities of infection and/or detection [18]. Detailed information on values used for scenario tree nodes are provided in Table II.

Bird group: The proportion of birds in the different bird groups ( $\mathrm{PrSwan}, \mathrm{PrD} / \mathrm{G} / \mathrm{M}$ and $\mathrm{PrOther}$ ) for September-April was calculated as the mean monthly count of birds in a group divided by the mean monthly total bird count, taken from the Lake Constance winter bird counts for September 2006-April $2007^{2}$. For May-August the bird group proportions were assumed to be those observed in the Lake Constance breeding bird count data for 2000 [3].

Unit infection status: The probability that a bird was infected $\left(P^{*} u\right)$ was defined as a set design prevalence. The design prevalence was $1 \%$ unless stated otherwise.

Bird dies: The probability that a bird died given that it was infected ( $P$ Dies $i$, where $i$ represents the Bird group Swan, $D / \bar{G} / \mathrm{M}$ or Other) was estimated from mortality rates observed in birds exposed to HPAI H5N1 [4, 5, 13-15, 20]. Uncertainty distributions for these parameters were increased in light of expert opinion expressed by EFSA [7] and wild bird AI surveillance findings in the EU in 2006 and 2007 $[10,11]$.

Test positive: Expert opinion was used to estimate the probability that an infected, sampled bird tested positive. Different estimates were used for live birds and dead birds (TestSe Dead or TestSe Live). Test specificity was assumed to be $100 \%$ as all positives would be subjected to confirmatory testing.

Bird sampled: Data on sampling numbers were obtained from the actual Constanze Project surveillance data. For Bycatch, Trap, Swan Catch and Hunt the mean monthly number of birds sampled was divided by the number of birds observed on Lake Constance. This gave the proportion of birds that were sampled (PSamp $\_$, where $i$ represents the Bird group).

\footnotetext{
${ }^{2}$ Unpublished data from the Swiss Ornithological Institute, Sempach, Switzerland.
}

Only samples taken around the lake shore should be included as the reference population counts were from the shore. However, recorded sampling locations for Hunt and Found dead were unreliable and uncertainty distributions of the mean monthly number of samples taken ( $N_{-}$Samp $i$ ) were created using the@RISK distribution Integer Uniform, from the number of samples reported as taken within $200 \mathrm{~m}$ of the shore to half of all samples taken.

To estimate the proportion of dead birds that were sampled by Found dead, the denominator was an estimate of the mean monthly number of birds that die in the reference population. This was derived as follows:

(1) Uncertainty distributions of normal yearly survival (Surv i) were estimated from literature [21]. The yearly survival distributions used for September-April and May-August were

$$
\begin{aligned}
& \text { Surv_Swan } \sim \operatorname{Uniform}(0.6,0.85), \\
& \text { Surv_D } / G / M \sim \operatorname{Uniform}(0.36,0.76) \text { and } \\
& \text { Surv_Other } \sim \operatorname{Uniform}(0.36,0.76) .
\end{aligned}
$$

(2) These were adjusted to provide monthly normal mortality estimates, thus:

$$
1-\text { Surv }_{-} i^{(1 / 12)} \text {. }
$$

(3) This was multiplied by the number of individuals in the bird group to estimate the number of deaths per month in a group under normal conditions.

The numerator (the mean monthly number of dead birds sampled) was divided by this estimated monthly number of deaths to give the proportion of dead birds that were sampled.

\subsubsection{Further Sentinel tree nodes}

The proportion of birds that visited the sentinel flocks was estimated from recorded waterbird visitors to the German sentinel flock between JanuaryDecember 2007. This was based on observations made for 30 min every 5 days by ornithologists running the sentinel facility ${ }^{3}$. The number of visitors to the Swiss and Austrian sentinels were each estimated by multiplying the number of visitors to the German

\footnotetext{
${ }^{3}$ Unpublished data from the Max Planck Institute for Ornithology, Radolfzell, Germany.
} 
flock by Uniform $(0.5,1.5)$. The number of visitors to all sentinels was summed and put as a proportion of all waterbirds on Lake Constance for the different Bird groups (PVisit_ $i$, where $i$ represents Bird group).

The probability that a bird infected the sentinel flock when it visited was derived from the opinions of two experts in avian influenza virology (PInf $i j$, where $i$ and $j$ represent Bird group and which expert respectively). For each bird group, each expert was asked to give the minimum, most likely and maximum probability that an infected bird transmitted infection to the sentinel flock when visiting; using these opinions, distributions were constructed (i.e. Betapert (minimum, most likely, maximum) - see Tab. II). When implementing the model experts were given equal weighting.

The probability of detecting infection in one or more sentinel birds given that infection had been transmitted to the sentinel flock (FlockTestSe) was estimated as follows:

(1) The test Sensitivity for one live bird tested was TestSe Live Uniform $(0.9,0.99)$.

(2) The Proportion of sentinel birds assumed to be infected at the time of testing given that the flock was infected, was PrInfect $\sim$ Uniform $(0.06,1)$, based on minimum and maximum values observed for all AI viruses from Swiss and Austrian sentinel data.

(3) Given that the number of sentinel birds sampled was 10 (the modal number of sentinel birds sampled), the probability that at least one sentinel bird was infected and tested positive was

$$
1-\left((1-(\text { TestSe_Live } \times \text { PrInfect }))^{10}\right) .
$$

Uncertainty relating to testing and transmission parameters was reflected in the scenario tree simulations.

\subsection{Cost of surveillance}

Direct cost estimates for running each SSC for one year were provided by Switzerland. Germany provided cost estimates for Sentinel, Trap, and Swan Catch. These estimates were adjusted to represent one month's surveillance. Where lacking, SSC cost for a country was assumed to be the mean cost for countries from which data was obtained (adjusted for the number of samples taken). See Table III for cost estimates.
Table III. Estimate of the mean monthly cost of AI surveillance in wild waterbirds on Lake Constance from September 2006 to August $2007(€)$. The cost is shown for the different Surveillance System Components (SSC) used.

\begin{tabular}{lcc}
\hline SSC & September-April & May-August \\
\hline Bycatch & 96 & 9 \\
Trap & 7047 & 7255 \\
Swan Catch & 3079 & 4070 \\
Found dead & 243 & 356 \\
Hunt & 874 & 0 \\
Sentinel & 7981 & 7981 \\
\hline
\end{tabular}

\subsection{Analysis}

\subsubsection{Component sensitivity}

For each SSC the probability of detection for one month's surveillance was calculated $(\mathrm{CSe})$. An estimate for the average month's surveillance between September-April was calculated, as well as for the average month's surveillance between May-August.

If the size of the reference population of wild birds was $n$ then $C S e$, the probability that at least one bird was infected, sampled and tested positive for Found dead was

$$
\begin{gathered}
1-\left(1-\sum_{i=1}^{3}\left(\text { Pr_i } i \times P^{*} u \times\right. \text { PDies_i }\right. \\
\times \text { PSamp_i } \times \text { TestSe_Dead }))^{n}
\end{gathered}
$$

where $i$ represents the Bird group.

The formula for CSe for SSC that sampled live birds (Bycatch, Hunt, Trap and Swan Catch) was

$$
\begin{aligned}
1- & \left(1-\sum_{i=1}^{3}\left(\operatorname{Pr}_{-} i \times P^{*} u \times\left(1-\text { PDies }_{-} i\right)\right.\right. \\
& \times \text { PSamp_i } i \times \text { TestSe_Live }))^{n} .
\end{aligned}
$$

For Sentinel, if SeSent $i$ was the probability of detecting one infected bird given it had not died, from Bird group $i$, then SeSent_ $i$ was

$$
\text { PVisits_ } i \times \text { Pinf_ } i \times \text { FlockTestSe. }
$$

For Bird group "Other" this was different for Mallards and NonMallards

$$
\begin{aligned}
\text { SeSent_Other }= & \sum_{i=1}^{2}\left(\operatorname{Pr}_{-} j \times \text { PVisits } j \times \operatorname{Pinf}_{-} j\right. \\
& \times \text { FlockTestSe })
\end{aligned}
$$


Table IV. Number of wild waterbirds sampled around Lake Constance by the different methods used for AI surveillance of wild waterbirds from September 2006 to August 2007. ( $D / G / M=$ diving ducks, grebes and mergansers).

\begin{tabular}{|c|c|c|c|c|c|c|c|}
\hline & \multicolumn{3}{|c|}{ September-April } & \multicolumn{3}{|c|}{ May-August } & \multirow[t]{2}{*}{ Total for year (all bird groups) } \\
\hline & Swan & $D / G / M$ & Other & Swan & $D / G / M$ & Other & \\
\hline Found dead & 19 & 12 & 35 & 8 & 6 & 52 & 132 \\
\hline Trap & 5 & 36 & 23 & 3 & 10 & 68 & 145 \\
\hline Hunt & 47 & 10 & 93 & 0 & 0 & 0 & 150 \\
\hline Swan Catch & 93 & 0 & 3 & 221 & 0 & 0 & 317 \\
\hline Bycatch & 0 & 11 & 11 & 0 & 0 & 1 & 23 \\
\hline
\end{tabular}

where $j$ represents "Mallard" or "Non_Mallard" and $\operatorname{Pr} j$ is the proportion of "Other" birds that are "Mallard" or "Non_Mallard". Given this, CSe is

$$
\begin{aligned}
1- & \left(1-\sum_{i=1}^{3}\left(\operatorname{Pr} \_i_{-} \times P^{*} u\right.\right. \\
& \left.\left.\left.\times\left(1-\text { PDies } \_i\right) \times \text { SeSent } \_i\right)\right)\right)^{n} .
\end{aligned}
$$

\subsubsection{Sensitivity analysis}

Design prevalence $\left(P^{*} u\right)$ values of $0.1 \%, 1 \%$ and $5 \%$ were evaluated separately. Changes in CSe Sentinel (September-April) caused by having half as many and a third as many wild bird visitors to sentinels (reducing PVisit_i) were examined. The effect of halving and doubling the number of birds sampled in September-April was investigated for SSC other than Sentinel. The effect of including all Hunt and Found dead samples instead of only those thought to come from the lake shore (and thus the reference population) was also investigated.

Multivariate stepwise regression was used to assess the variation in output $C S e$ caused by variation in the different input parameters (using @ RISK sensitivity analysis); normalised regression coefficients for each input are reported.

\subsubsection{Comparative sensitivity ratio (SR)}

Comparative sensitivity ratios, the ratio of one $\mathrm{CSe}$ to a reference, were calculated with the CSe for Trap as the reference. Thus $S R$ was

$$
\frac{C S e_{-} i}{C S e_{-} T r a p}
$$

CSe_Trap was chosen as the baseline as it had a narrow uncertainty distribution.

\subsubsection{Cost-effectiveness}

Cost-effectiveness was calculated as the mean cost per percent $\mathrm{CSe}$ for the different SSC as follows

$$
\frac{\mathrm{Cost} \_i}{\mathrm{CS} e_{-} i}
$$

where $i$ represents the different SSC, and CSe is given as a percentage.

Models were implemented in Excel (Microsoft Corporation) using@Risk (Palisade Corporation). Stochastic simulation with 10000 iterations for each model simulation was performed using Latin hypercube sampling with a randomly selected random number seed.

\section{RESULTS}

\subsection{Reference population and SSC samples}

The wild bird population counts used in the analysis are shown in Table II. Between September 2006-April 2007, 398 birds that were eligible for the study were sampled by SSC other than Sentinel. For May-August 2007, the equivalent figure was 369 samples (see Tab. IV for details of sampling). It was not possible to determine the SSC for four samples. Throughout the results the mode is reported with the 5th and 95th percentiles in brackets.

\subsection{Probability of detection and sensitivity analysis}

The probability of detecting HPAI H5N1 for the different SSC (CSe as a proportion not a percentage) is shown in Table $\mathrm{V}$. 
Table V. The probability of detection $(\mathrm{CSe})$ of HPAI H5N1 by different methods of wild waterbird surveillance, if infection was present in wild waterbirds on Lake Constance at $1 \%, 5 \%$ and $0.1 \%$ prevalence $\left(P^{*} u\right)$ from September 2006 to August 2007. Probabilities are shown as proportions and not percentages. The mode is shown with 5 th and 95 th percentiles in brackets.

\begin{tabular}{lcllllll}
\hline & $P^{*} u$ & & $1 \%$ & & $5 \%$ & & $0.1 \%$ \\
\hline September-April & Bycatch & 0.01 & $(0.01-0.02)$ & 0.06 & $(0.04-0.08)$ & 0.00 & $(0.00-0.00)$ \\
& Trap & 0.03 & $(0.02-0.04)$ & 0.12 & $(0.10-0.20)$ & 0.00 & $(0.00-0.00)$ \\
& Swan Catch & 0.00 & $(0.00-0.02)$ & 0.07 & $(0.02-0.09)$ & 0.00 & $(0.00-0.00)$ \\
& Found dead & 0.30 & $(0.14-0.53)$ & 0.95 & $(0.52-0.98)$ & 0.03 & $(0.01-0.07)$ \\
& Hunt & 0.01 & $(0.01-0.03)$ & 0.09 & $(0.03-0.15)$ & 0.00 & $(0.00-0.00)$ \\
& Sentinel & 0.73 & $(0.43-0.89)$ & 1.00 & $(0.94-1.00)$ & 0.12 & $(0.05-0.20)$ \\
& & & & & & \\
May-August & Bycatch & 0.00 & $(0.00-0.00)$ & 0.01 & $(0.00-0.01)$ & 0.00 & $(0.00-0.00)$ \\
& Trap & 0.08 & $(0.06-0.11)$ & 0.42 & $(0.28-0.45)$ & 0.01 & $(0.01-0.01)$ \\
& Swan Catch & 0.03 & $(0.01-0.08)$ & 0.32 & $(0.04-0.34)$ & 0.01 & $(0.00-0.01)$ \\
& Found dead & 0.37 & $(0.19-0.66)$ & 1.00 & $(0.64-1.00)$ & 0.04 & $(0.02-0.10)$ \\
& Hunt & 0.00 & $(0.00-0.00)$ & 0.00 & $(0.00-0.00)$ & 0.00 & $(0.00-0.00)$ \\
& Sentinel & 0.56 & $(0.33-0.80)$ & 1.00 & $(0.86-1.00)$ & 0.08 & $(0.04-0.15)$ \\
\hline
\end{tabular}

For September-April with $P^{*} u$ of $1 \%$, when the number of visitors to the sentinel flock was halved, the mode for $\mathrm{CSe}$ changed from 0.73 $(0.43-0.89)$ to $0.44(0.25-0.67)$, and when it was divided by three $C S e$ became 0.28 (0.17-0.52).

For other SSC, the probability of detection changed in an approximately linear fashion if the number of birds sampled was halved or doubled, i.e. sampling more birds increased the probability of detection, except for doubling the number of dead birds sampled, in which case the probability of detection less than doubled (data not shown).

When all samples were included, instead of only those thought to come from the shore, the CSe for Hunt was still zero in May-August and $0.06(0.05-0.08)$ in September-April. For Found dead it was $0.90(0.69-0.96)$ in MayAugust and $0.75(0.59-0.86)$ in SeptemberApril.

The regression sensitivity analysis (data not shown) revealed that in September-April, mortality rate in the bird group " $D / G / M$ " was the most influential parameter for Bycatch and Trap. In May-August mortality rate in the bird group "Other" was the most influential input for Bycatch and Trap, this parameter was the second most influential parameter for these SSC in September-April. Mortality rate of infected birds in the bird group "Swan" was perfectly negatively correlated with the probability of detection for Swan Catch for both September-April and May-August. For Found dead in September-April number of swans sampled had a coefficient of 0.75 and annual survival in swans had a coefficient of 0.38 . Found dead in May-August was influenced by the number of Other birds sampled and their annual survival. Hunt, only performed in September-April, was heavily influenced by the number of Other birds hunted. Sentinel was mostly influenced by whether opinion from Expert 1 or Expert 2 was chosen for the probability of transmission from a visiting bird to the sentinel flock, and by the mortality rate in Other birds.

\subsection{Comparative sensitivity ratio}

Looking at the mode (with the 5th and 95th percentiles in brackets) of the $S R$ of the SSC (the ratio of CSe $i$ to CSe Trap); Sentinel had the highest value, $19.9 \overline{7}(12.62-35.37)$ in September-April and $6.42(3.52-10.41)$ in May-August then Found dead with $S R=9.55$ (4.13-19.24) for September-April and 4.60 (2.03-8.36) in May-August. Swan Catch had a lower $S R$ than Bycatch in September-April, $0.19(0.11-0.72)$ versus $0.34(0.23-0.63)$ but 
Table VI. Cost-effectiveness of the different methods of surveillance for HPAI H5N1 in wild waterbirds on Lake Constance from September 2006 to August 2007; calculated as the mean monthly cost divided by the mean monthly probability of detection (Euros $/ \% \mathrm{CSe}$ ); mode with 5 th and 95 th percentiles in brackets are shown.

\begin{tabular}{lcccc}
\hline & \multicolumn{2}{c}{ September-April } & \multicolumn{2}{c}{ May-August } \\
\hline Found dead & 603 & $(507-1446)$ & 831 & $(531-1416)$ \\
Trap & 194285 & $(165000-336000)$ & 59071 & $(53100-93200)$ \\
Hunt & 50249 & $(41587-56021)$ & - & - \\
Sentinel & 9786 & $(8990-18600)$ & 11073 & $(10000-24400)$ \\
Swan Catch & 164231 & $(158000-857000)$ & 51736 & $(50400-476000)$ \\
Bycatch & 7340 & $(6050-11700)$ & 6248 & $(5540-10600)$ \\
\hline
\end{tabular}

in May-August this was reversed with Bycatch having a $S R$ of $0.013 \quad(0.01-0.02)$ and Swan Catch a $S R$ of $0.61(0.10-1.03)$.

\subsection{Cost-effectiveness}

Ratio of cost in Euros to percentage CSe when $P^{*} u$ was $1 \%$ is shown in Table VI. A high value represents poor cost-effectiveness. Found Dead was the most cost-effective. Trap was the least cost-effective.

\section{DISCUSSION}

With an assumed prevalence of HPAI H5N1 of $1 \%$ in waterbirds on Lake Constance, our results indicate that during winter months, surveillance using Bycatch, Trap, Swan Catch or Hunt would be very unlikely to detect infection. The probability of detection by Found dead was estimated to be between 0.14 and 0.53 and for Sentinel between 0.43 and 0.89. Similarly, for summer months, surveillance based on Found dead or Sentinel was more likely to detect HPAI H5N1 than any of the other strategies.

Even with a reduced number of bird visitors to sentinels the predicted $\mathrm{CSe}$ was still much higher than that estimated for other SSC except Found dead, confirming that the results are fairly robust against errors made in these estimates. It also suggests that a poorly placed sentinel, attracting fewer visitors, still has a relatively high probability of detecting HPAI
H5N1. Including all samples for Found dead and Hunt, and not just those from the lake shore, increased the $\mathrm{CSe}$, but did not alter conclusions drawn about which SSC had the highest probability of detecting infection.

No hunting was allowed in summer; hence the probability of detection by hunting at that time was zero. Swan catching was only conducted in August and September and therefore the probability of detection during other months would be zero. Averaging the probability of detection for September to April and May to August gives an overall measure of performance but hides any short term variation within these periods.

The sensitivity analysis indicated that Bycatch, Swan Catch and Trap CSe were heavily influenced by the level of mortality in HPAI H5N1 infected birds; when estimating mortality in the field from small, controlled studies, uncertainty is unavoidable. As the SSC sample only dead or only live birds, post-infection mortality is crucial. Obviously swan mortality is pivotal for swan catching and mortality in Mallards is important for sentinel surveillance as most sentinel visitors were Mallards. For sentinel, which of the two experts' opinion was used to estimate the probability of a visiting Mallard infecting the sentinel was the most influential parameter. This correctly reflects the uncertainty over this value.

The SR indicated that Trap was more likely to detect HPAI H5N1 than Hunt, Swan Catch and Bycatch; there was insufficient evidence to say that Sentinel had a greater chance of 
detecting HPAI H5N1 than Found dead. The population counts for wild birds are estimates; the potential for inaccuracies mean that caution should be exercised when making very precise comparisons between SSC. The fact that the $S R$ for both Found dead and Sentinel were smaller in May-August was in part due to the increased CSe of Trap in May-August and independent of the performance of Sentinel and Found dead. Due to lack of published data, the same value for estimated normal survival of wild birds was used for September-April and MayAugust, derived from annual survival estimates. This factor reduced the ability of the study to detect differences between these two time periods for Found dead surveillance, pushing estimates towards the yearly average.

Found dead had a high probability of detecting HPAI H5N1 infection because the probability of a dead bird being sampled by Found dead was much greater than the probability of a live bird being sampled via other SSC and the proportion infected was greater for dead birds than live birds. Swan Catch had the added problem that it targeted a bird species that has been shown to have a higher mortality rate $[5,14]$ and so were not available for live bird sampling. This was not an issue for Sentinel as it targeted Mallard visitors who often survived infection [4, 15] and thus were a good target for live bird sampling. In addition Sentinel sampled very large numbers of visiting birds and had a high probability of detecting infection in spite of the fact that detection required transmission to the sentinel. Bycatch sampled too few birds to have a high sensitivity.

Cost-effectiveness should be viewed together with $\mathrm{CSe}$ and total cost. For example, although Bycatch appeared to be cost-effective the CSe was poor and although Sentinel was fairly cost-effective it requires a relatively large investment. Swan Catch and Trap performed poorly on all three measures. Cost-effectiveness results apply to the specific surveillance performed and cannot be extrapolated; with different levels of expenditure cost-effectiveness will change. To predict cost-effectiveness at different sample sizes the complex relationships between cost and sample size, and sample size and sensitivity would have to be accounted for.
For example, Hunt may have fixed set-up costs, with extra costs varying linearly with the number of birds sampled; for live bird trapping set up costs could be high and then extra manning of a trap may increase sample size linearly until additional traps are required. Although sensitivity on the whole increased linearly with sample size this was due to the low sensitivities obtained. As the number of birds sampled increases, eventually additional sampling will yield diminishing additional sensitivity (as seen for Found dead), thus reducing the sensitivity per bird sampled and possibly cost-effectiveness. With additional economic data, curves plotting estimated cost against estimated sensitivity over a range of values for each SSC could be made to help select future surveillance strategies, although it should be noted that one trap may perform better than another due to factors other than cost, e.g. location.

The complexities of detecting infection by sentinel surveillance may be more accurately described by other modelling techniques; however, scenario tree analysis provides a transparent and consistent way of comparing the different SSC. Another factor in the interpretation of results for Sentinel is that it was assumed that 10 sentinel birds were always tested fortnightly. Although 10 was the most common number of birds tested, this did vary and on rare occasions none were tested. Testing 10 birds achieved good sensitivity, however, if no birds are tested the sensitivity will be zero. In suboptimal field conditions $\mathrm{CS}$ e may be more variable than described. Interpretation of sentinel HPAI serological tests was unclear and would provide minimal additional sensitivity as flock sensitivity was high based on RT-PCR alone.

A low design prevalence $\left(P^{*} u\right)$ was chosen in line with findings in wild birds $[10,11]$. The design prevalence assumes that there are a constant proportion of infected live animals and a fixed sub-group of dead animals of which a constant proportion are infected. During an outbreak the actual prevalence will vary, this is catered for by assessing performance with different design prevalences. Sentinel and Found dead had the highest probability of detection for all scenarios and at $0.1 \%$ design prevalence the probability of detecting 
HPAI H5N1 was less than 0.01 for all other SSC.

All Bird groups were assumed to have the same risk of infection. Although this may not be the case there is a lack of quantitative data required to assign different risks to the Bird groups. If during an outbreak the prevalence varied between bird groups it could alter the performance of the SSC depending on which group they target. Spatial clustering of disease on Lake Constance was not incorporated as the birds are not segregated to the same extend as animals on different farms [17]. Some degree of clustering may exist but is hard to define [9].

In early 2006, far more birds were reported by the public for Found dead sampling than subsequently, this was seen across Europe $[10,11]$. This period was deliberately excluded as it represents "abnormal" sampling activity done at an "abnormally" low cost. This may never be repeated. It highlights the lack of control that veterinary services have over sampling by Found dead and it would be interesting to consider the cost-effectiveness of active patrolling for dead birds.

A strength of Sentinel over Found dead is that it does not depend on HPAI H5N1 causing high mortality in order to detect it, in fact as live birds visit the sentinel, the lower the probability of an infected bird dying the greater the probability of detection; thus it can detect low pathogenic avian influenza (LPAI). On the other hand it does rely on infection occurring in Mallards, who were by far the most frequent visitor. To fully assess the value of the different SSC, their performance in surveillance of LPAI viruses should be considered. Hunt and Trap were thought to be an "effective method" for monitoring LPAI, with $2.7 \%$ of these samples LPAI positive ${ }^{4}$, however, Globig et al. believed that

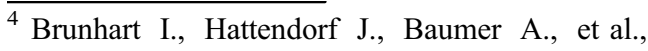
Investigation of the epidemiology of avian influenza in wild birds at Lake Constance - a Tri-lateral Project of Germany, Austria and Switzerland, in: Proceedings of the 12th Symposium of the International society for Veterinary Epidemiology and Economics, Durban, South Africa, 2009, pp. 274-276.
}

LPAI surveillance using live bird trapping was inferior to sentinel surveillance [8].

By having several SSC that perform well under different circumstances, overall surveillance system sensitivity will be maintained over a wider range of scenarios, the cost being the expense of maintaining several different SSC. It may be desirable to spend more to maximise sensitivity at perceived high risk periods and locations. Found dead by active patrols, Swan Catch, Hunt and Bycatch may provide useful periodic boosts to sensitivity. The value for money of these forms of surveillance is heavily dependent on the financial arrangements with those collecting the samples.

This study provides useful insights into the relative performance of the different SSC. However, when trying to generalise it must be remembered that in different settings, with different levels of sampling intensity, different wild bird populations and different costs, the results will vary.

In conclusion, Found dead seemed to be sensitive and highly cost-effective in a situation where mortality is high. Sentinel was also highly sensitive and reasonably cost-effective, and it allows the detection of AI when it causes low mortality as long as Mallards are infected. Although halving the number of dead birds sampled and sentinel visitors reduced the sensitivity of Found dead and Sentinel, they were still more sensitive than other SSC with all samples included. Live bird trapping had a low probability of detection and very low cost-effectiveness due to the small number of live birds sampled for the amount of expenditure. When trying to detect a disease that causes high mortality, live bird sampling may be ineffective unless the sample size is large.

Acknowledgements. Thanks to Christian Griot and Anette Baumer from the Institute of Virology and Immunoprophylaxis, Mittelhäusern, Switzerland and Anja Globig and colleagues at the Friedrich-Loeffler-Institute, Insel Riems, Germany for data provision and expert opinion on model parameters. We also thank: All participants in the Constanze Project, Iris Brunhart and Annette Sauter, Federal Veterinary Office, Bern, Switzerland and Wolfgang Fiedler, Max Planck Institute for Ornithology, Radolfzell, Germany for surveillance and ornithological advice. Theodore Knight-Jones is part funded by The Royal College of Veterinary Surgeons Trust. 


\section{REFERENCES}

[1] Alexander D.J., A review of avian influenza in different bird species, Vet. Microbiol. (2000) 74:3-13.

[2] Alexander D.J., An overview of the epidemiology of avian influenza, Vaccine (2007) 25:5637-5644.

[3] Bauer H.G., Heine G., Bodensee - Brutvogelatlas 2000, Ornithologische Arbeitsgemeinschaft Bodensee, 2005 .

[4] Brown J.D., Stallknecht D.E., Beck J.R., Suarez D.L., Swayne D.E., Susceptibility of North American ducks and gulls to H5N1 highly pathogenic avian influenza viruses, Emerg. Infect. Dis. (2006) 12:16631670.

[5] Brown J.D., Stallknecht D.E., Swayne D.E., Experimental infection of swans and geese with highly pathogenic avian influenza virus (H5N1) of Asian lineage, Emerg. Infect. Dis. (2008) 14:136-142.

[6] De Jong M.D., Hien T.T., Avian influenza A (H5N1), J. Clin. Virol. (2006) 35:2-13.

[7] EFSA, Scientific Opinion of the Panel on Animal Health and Welfare on a request from the European Commission on Animal health and welfare aspects of avian influenza and the risk of its introduction into the EU poultry holdings, The EFSA Journal (2008) 715:1-161.

[8] Globig A., Baumer A., Revilla-Fernández S., Beer B., Wodak E., Fink M., et al., Ducks as sentinels for avian influenza in wild birds, Emerg. Infect. Dis. (2009) 15:1633-1636.

[9] Happold J.R., Brunhart I., Schwermer H., Stark K.D., Surveillance of $\mathrm{H} 5$ avian influenza virus in wild birds found dead, Avian Dis. (2008) 52:100-105.

[10] Hesterberg U., Harris K., Stroud D., KnightJones T., Cook A., Brown I., Annual report on surveillance for avian influenza in wild birds in the EU during 2007 (SANCO/2181/2008 - Rev.1), DG-SANCO surveillance report, 2008.

[11] Hesterberg U., Harris K., Stroud D., Guberti V., Busani L., Pittman M., et al., Avian influenza surveillance in wild birds in the European Union in 2006, 2009, pp. 1-14.

[12] Homedes N., The disability-adjusted life year (DALY) definition, measurement and potential use by Nuria Homedes, World Bank, Washington, D.C., 1996.

[13] Isoda N., Sakoda Y., Kishida N., Bai G.R., Matsuda K., Umemura T., Kida H., Pathogenicity of a highly pathogenic avian influenza virus, A/chicken/ Yamaguchi/7/04 (H5N1) in different species of birds and mammals, Arch. Virol. (2006) 151:1267-1279.

[14] Kalthoff D., Breithaupt A., Teifke J.P., Globig A., Harder T., Mettenleiter T.C., Beer M., Highly pathogenic avian influenza virus (H5N1) in experimentally infected adult mute swans, Emerg. Infect. Dis. (2008) 14:1267-1270.

[15] Keawcharoen J., van Riel D., van Amerongen G., Bestebroer T., Beyer W.E., van Lavieren R., et al., Wild ducks as long-distance vectors of highly pathogenic avian influenza virus (H5N1), Emerg. Infect. Dis. (2008) 14:600-607.

[16] Kilpatrick A.M., Chmura A.A., Gibbons D.W., Fleischer R.C., Marra P.P., Daszak P., Predicting the global spread of H5N1 avian influenza, Proc. Natl. Acad. Sci. USA (2006) 103:19368-19373.

[17] Martin P.A., Cameron A.R., Barfod K., Sergeant E.S., Greiner M., Demonstrating freedom from disease using multiple complex data sources 2: case study classical swine fever in Denmark, Prev. Vet. Med. (2007) 79:98-115.

[18] Martin P.A., Cameron A.R., Greiner M., Demonstrating freedom from disease using multiple complex data sources 1: a new methodology based on scenario trees, Prev. Vet. Med. (2007) 79:71-97.

[19] Olsen B., Munster V.J., Wallensten A., Waldenstrom J., Osterhaus A.D., Fouchier R.A., Global patterns of influenza a virus in wild birds, Science (2006) 312:384-388.

[20] Pasick J., Berhane Y., Embury-Hyatt C., Copps J., Kehler H., Handel K., et al., Susceptibility of Canada geese (Branta canadensis) to highly pathogenic avian influenza virus (H5N1), Emerg. Infect. Dis. (2007) 13:1821-1827.

[21] Schekkerman H., Slaterus R., Population dynamics and prevalence of influenza A viruses in mallard, mute swan and other wildfowl, in: Avian influenza and wildfowl populations, British Trust for Ornithology (BTO), The Nunnery, Thetford, Norfolk, 2008.

[22] Stallknecht D.E., Shane S.M., Host range of avian influenza virus in free-living birds, Vet. Res. Commun. (1988) 12:125-141.

[23] Webster R.G., Bean W.J., Gorman O.T., Chambers T.M., Kawaoka Y., Evolution and ecology of influenza A viruses, Microbiol. Rev. (1992) 56:152179 . 